\title{
RESEARCH REPORT EFFECTIVENESS OF ISCHEMIC COMPRESSION ON TRIGGER POINTS FOR REDUCTION OF PAIN AND SPASM OF TRAPEZIUS MUSCLE
}

\begin{abstract}
Myofascial trigger point is a hyperirritable nodule present in a palpable taut band of skeletal muscle, often results from muscle injury or repetitive strain that cause pain and tightness. Myofascial trigger points are one of the most common causes of chronic neck pain. This study aims to determine the efficacy of ischemic compression in comparison with myofascial stretching on trigger points of trapezius muscle for reduction of pain and spasm. Randomized Control Trial. The study was conducted in Ziauddin Hospital. 96 participants were enrolled in the study. Participants were divided into two groups equally and randomly, Group (A) an intervention group treated with hot pack, ultrasound therapy and ischemic compression, Group (B) a control group treated with hot pack, ultrasound therapy and myofascial stretching. This regime was followed thrice a week for three weeks. Statistically significant $(P<0.05)$ changes in the values were found in Group A and Group B for Visual Analog scale and Penn spasm frequency scale post treatment. The results showed that there is significant difference found after both interventions for the treatment of pain and spasm caused by myofascial trigger point. It cannot be said that ischemic compression is more effective than myofascial stretching for the treatment of myofascial trigger points of trapezius muscle.
\end{abstract}

\section{KEYWORDS}

Myofascial trigger point, Trapezius muscle spasm, neck pain, ischemic compression, manual therapy, myofascial stretching.

\author{
Mubarra Rao \\ Physiotherapist \\ Ziauddin University \\ mubarrar@gmail.com \\ Sadia Shafaq \\ Lecturer \\ Ziauddin College of Rehabilitation Sciences \\ Ziauddin University \\ Dr.sadiarana.who@gmail.com
}

[Rao M, Shafaq S. Effectiveness of Ischemic Compression on Trigger Points for Reduction of Pain and Spasm of Trapezius Muscle. Pak. j. rehabil. 2018;7(1):21-27] 


\section{INTRODUCTION}

It is reported that the neck pain in adults has a prevalence of about $30 \%$ to $50 \%$. Myofascial trigger points play a big role in causing restricted movement, pain and functional limitation. In the origination of neck pain, it is clinically evident that myofascial trigger point plays a significant role. Myofascial trigger points are $20 \%$ prevalent in right upper trapezius muscle. The upper trapezius is often the muscle of interest in investigation of trigger points ${ }^{1-4}$. The upper trapezius muscle is more prone to myofascial trigger points which are highly localized intense taut band of skeletal muscles 5,6 . The existence of myofascial trigger points in upper trapezius muscle is one of the most typical finding in individuals with neck pain ${ }^{7}$. Anyone at any time during their lifespan can experience myofascial trigger point. It is nearly a stressful though not life threatening event in a person's life ${ }^{8}$. The patho physiology of its creation is not clear enough and modern research recommends that there is a low oxygen and nutrition in injured muscles fibers that automatically causes muscle contraction?. The pain of trapezius muscle trigger point is felt over the base of the neck posteriorly leading towards the shoulder ${ }^{10}$. Myofascial trigger point formation and its activation are dependent on postural stress ${ }^{11}$. Myofascial trigger points are classified on the basis of pain into active and latent. Active trigger points are a clinical pain symptom (spontaneous pain). Conversely latent myofascial trigger point are non-spontaneous i.e. pain is felt only on palpation/compression. Diagnosis of myofascial trigger point can be done through physical examination or through different devic$e^{12,13}$. Myofascial trigger points can be studied under ultrasound in relation to physical examination $^{14}$.

For the diagnosis of myofascial trigger points, essential and confirmatory criteria that comprises of four elements referring to the physical signs found in myofascial trigger point, taut band, tenderness, jump sign, referred pain and restricted range of motion, can be used ${ }^{15,16}$.

'The key to treating trigger points is to lengthen the muscle fibers that are shortened by the trigger point mechanism'16. There are several methods of treatment such as massage, stretching, ultrasound therapy, muscle energy techniques, dry needling, local injections, integrated neuromuscular inhibition technique and ischemic compression 12,17,18. Through these treatments the blood circulation is enhanced, lengthening of the sarcomere, ultimately it increases the range of motion and reduce the muscle tension thus releasing the spasm, relieving pain ${ }^{11,17}$.

Modern record analysis of physio-therapeutic treatment for myofascial trigger points decided that the most frequently used techniques for short term pain relief were trigger point pressure release, ischemic compression. Evidence supports that ischemic compression in a short span of time showed significant improvement in pain and range of motion in office workers with neck pain ${ }^{19}$.

The procedure of Ischemic compression produces local ischemia to muscle, tissue gets recovery by re perfusion thereby relieving transient blood flow occlusion ${ }^{4,20}$. The aim of this study was to determine the efficacy of ischemic compression in comparison with myofascial stretching on trigger points of trapezius muscle for the reduction of pain and spasm.

\section{METHODOLOGY}

Randomized Control Trial was conducted in Ziauddin Hospital, Karachi. The sample size of 96 participants comprising of 40 males and 56 females were randomly enrolled into two groups using the chit box method. The two groups were named A and B, each comprising of 48 participants. The study was conducted over 3 weeks in 9 sessions (thrice weekly).

\section{Procedure}

The purpose and procedure of the study was verbally explained to the participants and consent was taken. Physical therapist performed baseline assessment on the 1st day (pre-test score) using the outcome measure, visual analog scale, spasm frequency scale and severity of spasm. Group A was treated with, hot pack, ultrasound and ischemic compression. Hot pack was stored in hydro collator tank of $74.5-80^{\circ} \mathrm{C}$ for 30 minutes), all the participants were instructed to lie down in prone position with neck in neutral position. The hot pack was covered in 6 layers of towel and then applied over the myofascial trigger points for 20 minutes. Ultrasound therapy was applied over the area of myofascial trigger point for 10 minutes, with unfocused beam, frequency $1-3 \mathrm{MHz}$, Intensity $0.5-2.0$ W/cm2, Duty cycle 100\%, continuous bursts, followed by ischemic compression (compressed it with $2-4 \mathrm{~kg} / \mathrm{cm} 2$ pressure for 10 to 20 seconds) $)^{21-23}$. Group B was treated with traditional physical therapy treatment including hot pack, ultrasound therapy (same protocols as group A), followed by myofascial stretching (neck flexion, side flexion towards the opposite side (unaffected side) and then slightly rotated towards the affected side, position was held for 20 seconds $)^{16}$. Both the groups were treated thrice a week (alternate days) for 3 weeks. After the treatment regime was completed participants were reassessed (post-test score) via Visual Analog Scale and Penn Spasm Severity Scale.

\section{Inclusion criteria}

- Both the genders aged between 20-40 years.

- Pain or spasm in trapezius muscles. 
- Presence of palpable taut band.

- Pain pattern recognition by patient.

- Visual or tactile indication of latent trigger point.

- On manual pressure pain or altered sensation.

- Delicate spot tenderness of a nodule.

- Painful or limited end range of motion.

\section{Exclusion criteria}

- On medication other than non-steroidal anti-inflammatory drugs for pain.

- Muscular pathology.

- Cognitive deficit.

- Having any severe health problem such as uncontrolled hypertension or psychological issues.

\section{Data analysis}

Data analysis was performed using SPSS. The pre and posttest mean and standard deviation with P-value was calculated for both the groups with Paired T-test. In order to determine whether there were differences $(\mathrm{P}<.05)$ between the two groups on the posttest scores, an Independent T-test was performed. Level of significance for whole statistical analysis was kept at $5 \%$.

\section{RESULTS}

A total of 96 participants were enrolled in the study. There were 40 males (38\%) and 56 females (58\%) shown in chart-1. Each group had 48 participants. The average age of the participants was $32.3 \pm 5.6$ years. In experimental group (group A) there were 31 females and 17 males and in control group (Group B), 25 females and 23 males.

Mean and standard deviation of the pretest and posttest assessment of outcome measures was calculated. Visual analog scale showed significant decrease in posttest mean value. Spasm frequency scale also showed significant decrease in posttest mean value. However, severity of spasm in experimental group showed significant decrease in posttest mean value while it showed no significant decrease in posttest mean value of control group (Table 1).

Independent T-test used to determine significant difference between two groups. The posttest analysis indicated significant changes in the symptoms among both groups i.e. $\mathrm{P}<0.005$ (Table 2). Establishing that ischemic compression and myofascial stretching are both equally effective in treating the myofascial trigger points of trapezius muscle.

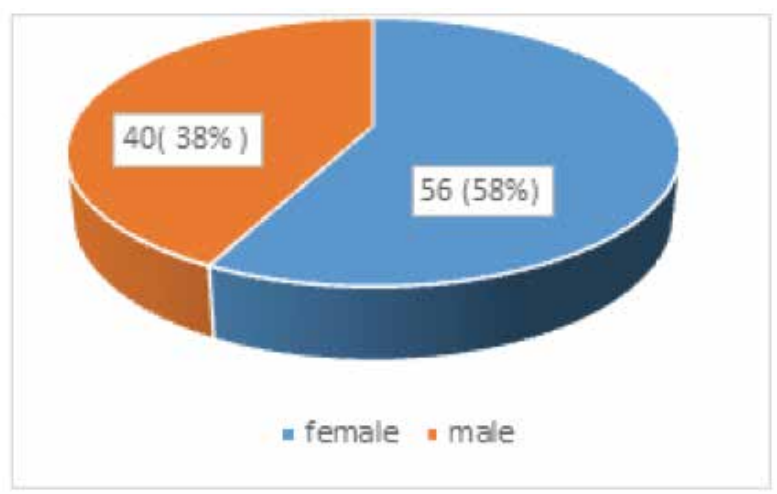

\section{Chart-1 Gender Distribution (frequency)}

Table 1: Pre and Post intervention readings of Group A \& B

\begin{tabular}{|l|l|l|l|l|}
\hline \multirow{4}{*}{ Group } & & Pre & Post & $\begin{array}{l}\text { P- } \\
\text { Value }\end{array}$ \\
\cline { 2 - 5 } A & VAS & $\begin{array}{l}3.19 \\
\pm 0.77\end{array}$ & $\begin{array}{l}1.76 \\
\pm 0.78\end{array}$ & 0.001 \\
\cline { 2 - 5 } & SFS & $\begin{array}{l}7.20 \\
\pm 1.18\end{array}$ & $\begin{array}{l}3.94 \\
\pm 1.26\end{array}$ & 0.000 \\
\cline { 2 - 5 } & SOS & $\begin{array}{l}2.69 \\
\pm 0.47\end{array}$ & $\begin{array}{l}1.20 \\
\pm 0.41\end{array}$ & 0.016 \\
\hline \multirow{4}{*}{\begin{tabular}{l}
04 \\
Group \\
\cline { 2 - 5 } B
\end{tabular}} & VAS & $\begin{array}{l}3.54 \pm \\
0.77\end{array}$ & $\begin{array}{l}1.29 \\
\pm 0.54\end{array}$ & 0.008 \\
\cline { 2 - 5 } & SFS & $\begin{array}{l}7.02 \\
\pm 1.39\end{array}$ & $\begin{array}{l}2.81 \\
\pm 0.79\end{array}$ & 0.004 \\
\cline { 2 - 5 } & SOS & $\begin{array}{l}2.79 \\
\pm 0.41\end{array}$ & $\begin{array}{l}1.41 \\
\pm 0.49\end{array}$ & 0.123 \\
\hline
\end{tabular}

Table 2: T-test for equality of means

\begin{tabular}{|c|c|c|c|c|c|}
\hline \multicolumn{6}{|c|}{ Table 2: T-test for equality of means } \\
\hline & $\mathrm{t}$ & $\mathrm{df}$ & $\begin{array}{c}\text { Sig } \\
\text { (2-tail) }\end{array}$ & $\begin{array}{c}\text { Mean } \\
\mathrm{df}\end{array}$ & $\begin{array}{c}\text { Std. } \\
\text { Error } \\
\mathrm{df}\end{array}$ \\
\hline VAS & 2.893 & 94 & 0.005 & 0.40 & 0.14 \\
\hline SFS & 5.237 & 94 & 0.000 & 1.13 & 0.21 \\
\hline SOS & -2.236 & 94 & 0.028 & -0.21 & 0.10 \\
\hline
\end{tabular}




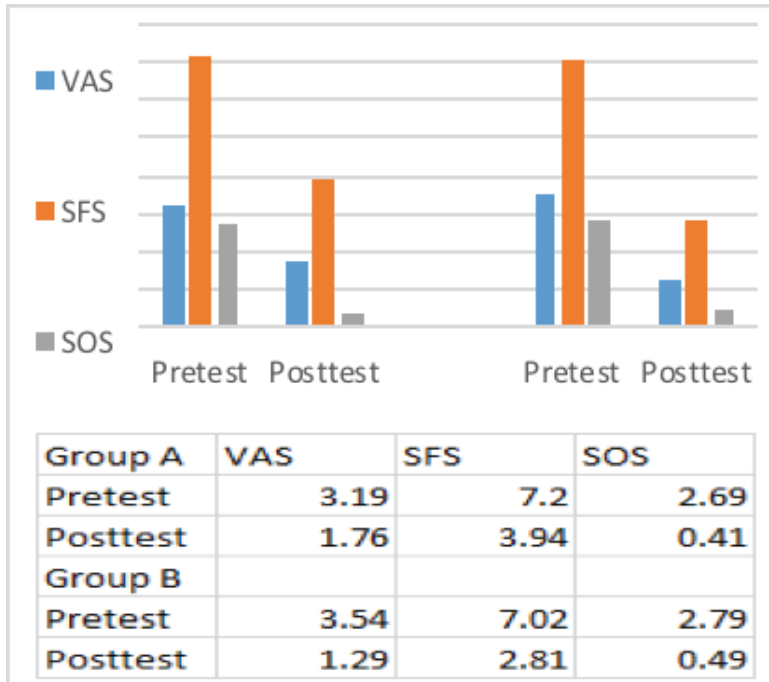

Graph 1: pretest and posttest readings (mean) of Group A and Group B.

\section{DISCUSSION}

The focus of this study was to evaluate the effectiveness of ischemic compression in comparison with myofascial stretching for treatment of myofascial trigger points of trapezius muscle. This was in accordance with Travell who stated that ischemic compression reduces the sensitivity of trigger points ${ }^{13}$. Outcome measures were visual analog scale for the pain and Penn spasm frequency scale for the frequency and severity of spasm. Our study showed statistically significant difference between pre-test and post-test results in both groups respectively. However in a study conducted by Shah et al, the pre and post scores of VAS, PPT and CROM (lateral flexion of the opposite side) in 30 patients (with nonspecific neck pain) were studied. After a regime, consisting of Ischemic compression or muscle energy technique along with isometric exercises for neck and stretching exercises of upper trapezius muscle, for 1 week was followed, no significant difference( $\mathrm{P}=0.332)$ in mean VAS score between group A and Group B was found, furthermore, the mean PPT score between Group A and B was found to be significant ( $P$ is 0.002$)$ and the mean CROM score between Group A and B was also found to be significant $(P<0.01)$. Results showed a significant improvement in the pressure pain threshold and range of motion after the application of ischemic compression in comparison with muscle energy technique ${ }^{23}$.

Gemmel et al, compared the ischemic compression trigger point pressure release and sham ultrasound therapy on 45 subjects (with nonspecific neck pain of at least 3 months and pain of at least $30 \mathrm{~mm}$ on VAS) and found only a slight reduction of symptoms in all the groups which was not significant. Mean Visual Analog Scale $(P=0.5721)$, mean
Pressure Pain Threshold $(P=0.2171)$ and mean Cervical range of motion scores $(P=0.8805)$ were found to be not significant, However it was found that ischemic compression showed significantly better results than sham ultrasound therapy ${ }^{24}$. However in our study improvement in symptoms were found statistically significant in both groups $(\mathrm{P}<$ 0.05).

Hains et al, in their study, assessed the Shoulder Pain and Disability Index (SPADI) score before and after ischemic compression was applied on trigger points in chronic shoulder pain patients. After the first 15 treatments as compared to control group, experimental group showed a significant decrease in SPADI scores ( $P=.003)$. After 6 months, the decrease in SPADI scores still existed (P.001).This study reported that ischemic compression showed significant changes in the SPADI score ${ }^{25}$. On the contrary, our study assessed the visual analog scale and Penn spasm frequency scale before and after application of ischemic compression which showed significant change $(P<0.05)$.

Gulick et al, in their study of 28 subjects with trigger points in upper musculature, reported significant difference in pretest and posttest sensitivity of myofascial trigger point in patients treated with ischemic compression in comparison with patients who were not treated with ischemic compression $(p=0.04)^{26}$. Whereas, in our study, patients treated with ischemic compression as well as myofascial stretching showed significant decrease in pain threshold $(P<0.05)$.

Paz et al, reports that two researches of high quality, namely, Hains et al and Bron et al, provides evidence that patients with chronic pain in shoulder(myofascial trigger points) can be treated with ischemic compression. One study reported the reduction in SPADI score and the other study after 12 week treatment reported clinically significant results $(p<0.05)$ respectively ${ }^{27}$. Similarly in our study, decrease in VAS and PENN scale pre-test and posttest was observed (significant $\mathrm{P}<0.05$ ).

Hanten et al evaluated the effectiveness of ischemic compression and stretching as a combination therapy for the treatment of myofascial pain syndrome on neck and lower back region, this study showed significant improvement in pain threshold. Our study evaluated the effectiveness of ischemic compression in comparison with myofascial stretching for the treatment of myofascial trigger points in trapezius muscle. The results in our study were found to be significant in both interventions $(P<0.05)^{28}$.

Study by Ganesh et al provided evidence about ischemic compression and mobilization, both has significant role in releasing upper trapezius trigger points. Using ANOVA, assessed the pretest and posttest scores of both the experimental groups as compared to the control group and found statisti- 
cally and clinically significant improvement in experimental groups $(p<0.05)$. But the effect size between both the experimental groups was small $(<0.3)^{29}$. This study is in accordance with our study for the use of ischemic compression, however the results in our study shows significant changes after both interventions $(P<0.05)$.

Cagnie et al in his study reported that general pain (neck/shoulder), pressure pain perception (( $\mathrm{P}=$ $.001)$, range of motion significantly improved post ischemic compression treatment but NDI score showed no significant improvement ${ }^{30}$. In our study, post ischemic compression as well as myofascial stretching shows significant pain reduction and decreased severity of trigger point. The end result was statistically significant in both groups $(P<0.05)$. Kannan et al compared ischemic compression, laser therapy and ultrasound therapy in a sample size of 45 . Through ANOVA he reported that all three interventions showed significant improvement statistically $(p<0.05)$ pre and post-trial ${ }^{31}$. Our study used ischemic compression as the main intervention and ultrasound as a protocol. Results were significant ( $P$ $<0.05)$.

Ravichandran et al, in their study (30 subjects) reported that between the control and experimental group, there was no significant difference $(p \geq$ 0.05 ) but range of motion improved in experimental group and within group data showed high statistical significance $(p \leq 0.01)^{7}$. On the contrary, in our study there was significant difference in pain and spasm severity in both control and experimental group $(P<$ 0.05), however range of motion was not an outcome measure in our study.

Abdelhamid et al reported that ischemic compression is more effective in improving trigger point symptoms in comparison with traditional physical therapy treatments. In their study they assessed the VAS, NPAD and CROM among 40 patients with mechanical neck pain. Posttest assessment showed statistically significant difference from pretest assessment in VAS, NPAD and CROM ${ }^{19}$. Conversely in our study ischemic compression was found to be not superior to myofascial stretching.

Kim et el, reported that ischemic compression with trigger point injection showed significantly better results than only trigger point injection. It was also reported that duration of ischemic compression (30 seconds or 60 seconds) did not affect the end results 17. Contrary to this, our study applied ischemic compression along with hot pack and ultrasound therapy and the duration for IC was 10 to 20 seconds. Contrary to Kim et al study, Sedgh et al reported that IC with duration of 90 seconds showed better results than IC in 30 seconds $^{12}$.

Menaka et al reported that ischemic compression followed by stretching improved the trigger point symptoms significantly. In their study they assessed pain intensity using Numerical Pain Rating Scale (NPRS) and PPT using pain algometer. There was statistically significant difference found between the pretest scores and posttest scores ${ }^{8}$. Whereas in our study we assessed pain intensity using VAS and severity of spasm using Penn scale in comparison between ischemic compression and myofascial stretching which showed significant changes in both groups $(P<0.05)$.

In our study, we used hot pack therapy and ultrasound therapy as protocols for both groups. The use of ultrasound therapy was supported with study by Draper Do et al, in which he states that the thermal effects of ultrasound reduced the stiffness of myofascial trigger points ${ }^{32}$. Khan AA et al states that ultrasound therapy improves the circulation and pain ${ }^{10}$. The use of hot pack therapy was supported with the study by Dararat et al, who states that hot pack therapy enhances circulation of the affected tissue ${ }^{15}$.

In the study by llter et al, through investigation, they reported that in comparison with pulsed ultrasound, continuous ultrasound showed significantly more improvement $(P=0.05)^{33}$. This study supports the use of ultrasound as a protocol in our study. Another study by Kavadar et al, provides evidence that ultrasound provides significantly $(p<0.001)$ good results ${ }^{34}$. The study by Benjaboonyanupap et al reported that hot pack therapy along with ultrasound therapy can be used for the treatment of myofascial trigger points as they induce physiological changes ${ }^{15}$.

To evaluate the effectiveness of ischemic compression, we compared its effects with the effects of myofascial stretching. Though the use of myofascial stretching was not supported with studies, as there is a lack of literature availability regarding myofascial stretching. However, Kostopoulos et al, in the book, mentioned the use of myofascial stretching for the treatment of myofascial trigger points. More research needs to be conducted for the beneficial use of myofascial stretching. Kostopoulos et al, compared effectiveness of ischemic compression in combination with passive stretching and noted that it was significantly more effective than other treatment options ${ }^{15}$. Also, evidence by Cagnie B et al showed that ischemic compression increased range of motion and decreased pain in the management of trigger point ${ }^{35}$. Similarly in our study, ischemic compression decreased the pain, which was found to be statistically significant $(P<0.05)$.

Nambi et al, in the study assessed VAS and ROM after treating trigger points with ischemic compression (group A) and muscle energy technique (Group B). No significant ( $P>0.05$ ) changes were found in Vas score between group $A$ and group $B$ whereas statistically significant $(P<0.05)$ changes 
were found in ROM between group A and group B. Group B i.e. muscle energy technique showed better results than ischemic compression. This study concludes that ischemic compression and muscle energy technique can be used as intervention in trigger point release to improve ROM and decrease pain but MET along with ultrasound showed better results ${ }^{36}$. In our study ischemic compression in comparison with myofascial stretching showed statistically significant results in both groups $(P<0.05)$.

Our study didn't focus on the range of motion restriction due to myofascial trigger points. Travell and Simmons et al states that range of motion can be restricted due to trigger points but only a handful of studies support the co relation between myofascial trigger points and decrease in range of motion $^{13}$.

The study was conducted in a short time period which is one of limitations of this study. Other limitations were, our study only focused on the pain intensity and severity of spasm. More outcome measures and variables can be assessed. There is more room for further research. Our study was focused on ischemic compression and not on myofascial stretching. There seem to be lack of clinically evident research regarding the use of myofascial stretching, so this can be a topic for further research.

\section{CONCLUSION}

The results showed that ischemic compression have more significant effect in reduction of symptoms of myofascial trigger point, as significant difference was found for the effectiveness of ischemic compression in comparison with myofascial stretching of trapezius muscle for the treatment of pain and spasm caused by myofascial trigger point.

\section{REFERENCES}

[1] Lobo CC, Costa SPD, Herranz EH. Efficacy of Deep Dry Needling on Latent Myofascial Trigger Points in Older Adult with Nonspecific Shoulder: a randomized, controlled clinical trial .J Geriatr Phys Ther 2017;40(2):63

[2] Cagnie B, Dhooge F, Van Akeleyen J, Cools A, Cambier D, Danneels L. Changes in microcirculation of the trapezius muscle during a prolonged computer task. Eur J Appl Physiol 2012;112(9):3305-3312.

[3] Shin SJ, Yoo WG. Changes in cervical range of motion, flexion-relaxation ratio and pain with visual display terminal work. J Occup Health. 2014;47(2):261-5.

[4] Grieve R, Barnett S, Coghill N, Cramp F. The prevalence of latent myofascial trigger points and diagnostic criteria of the triceps surae and upper trapezius: a cross sectional study. Physiotherapy. 2013;99(4):278-84.

[5] Hesari S, Attarbashi-Moghadam B, Shadmehr A. Comparison of Dry Needling and Physical Therapy in Patients with Trapezius Myofascial Pain Syndrome. J Mod Rehab 2016; 10(1):43-47.

[6] Castelein B et al. (2016) Are chronic neck pain, scapular dyskinesis and altered scapulothoracic muscle activity interrelated? A case-control study with the surface and finewire EMG. J Electromyogr Kinesiol. 2016; 31:136-143.

[7] Ravichandran P, Ponni K, Aseer AL.Effectiveness of ischemic compression on trapezius myofascial trigger points in neck pain. Int J Physiother. 2016;3(2):186-192.

[8] Menaka T, Kalaichandran A. Effect of Ischemic Compression Followed by Stretching on Myofascial Trigger Points. International Journal of Scientific and Research Publications. 2015;5(1):1-6.

[9] Akamatsu FE, Saleh S, Pinesi HT, Rodrigues KR, Zandoná CB, Andrade M, Jacomo AL. Anatomical basis of the myofascial trigger points of the trapezius muscle. Int. J. Morphol. 2013;31 (3):915-920.

[10] Khan AA, Farooqui SI, Sumble S, Khan MS. Efficacy Of Deep Friction Massage And Ultrasound In The Treatment Of Upper Trapezius Spasm- A Randomized Control Trail. JMDSR.2015;2(12):30-34.

[11] Zaky LA, Rashad GM. Efficacy of Ischemic Compression Followed by Exercises Therapy versus Rehabilitation Program in Treatment of Postural Scoliosis. Bull. Fac. Ph. Th. Cairo Univ.2013; 18(1). Sedgh E, Okhovatian F, Naimi SS, Baghban AA.

[12] Comparison of the Immediate Effects of Various Durations of Trigger Point Compression Technique on Latent Trigger Points of the Upper Trapezius Muscle. J Clin Physio Res. 2016;1(2): 49-53.

[13] Travell JG, Simons DG. Myofascial pain and dysfunction - The trigger point manual. 2nd ed.Baltimore: Lippincott Williams \& Wilkins; 1998.

[14] Jafari, M, Bahrpeyma F, Togha M, Effect of ischemic compression for cervicogenic headache and elastic behavior of active trigger point in the sternocleidomastoid muscle using ultrasound imaging, J Bodyw Mov Ther.2017 ;21 (4):933-9.

[15] Benjaboonyanupap D, Paungmali A,Pirunsan U.Effect of Therapeutic Sequence of Hot Pack and Ultrasound on Physiological Response Over Trigger Point of Upper Trapezius. Asian J Sports Med. 2015;6(3): 57-61.

[16] Kostopoulos D, Rizopoulos K. The manual of trigger point and myofascial therapy. Astoria, New York: Slack; 2001.

[17] Kim SA, Oh KY, Choi WH, Kim IK.Ischemic Compression After Trigger Point Injection Affect the Treatment of Myofascial Trigger Points. Ann Rehab Med.2013;37(4):541-546

[18] Saadat Z, Hemmati L, Pirouzi S, Ataollahi M, 
Ali-mohammadi F. Effects of integrated Neuromuscular Inhibition Technique on pain threshold and pain intensity in patients with upper trapezius trigger points, J Bodyw Mov Ther.2018;22(4): 937-940

[19] Abdelhamid ASM, Balbaa AAH, koura G, Elshiwi AMF, Abdelaal AAM. Ischemic Compression versus Traditional Physical Therapy in Treatment of Chronic Mechanical Neck Pain. Int J Adv Res.2015;3(1): 931-938.

[20] Gatterman MI, McDowell BL. Whiplash: A patient-centered approach to management.1st edition. St. Louis, Missouri: Elsevier; 2012.

[21] Belanger AY. Therapeutic electro physical agents.3rd edition. Quebec (Canada): 2014.

[22] Jaeger B. Myofascial trigger point pain. Alpha Omegan. 2013;106(1-2):14-22.

[23] Shah N, Shah N. Comparison of two treatment techniques: Muscle energy technique and Ischemic compression on upper trapezius trigger point in subjects with non- specific neck pain. Int J Rehabil Res. 2015;4(5):260-264.

[24] Gemmell H, Miller P, Nordstrom H.Immediate effect of ischemic compression and trigger point release on neck pain and upper trapezius trigger points: A randomized control trial. Clin Chiropr. 2008; 11 (1):30-6.

[25] Hains G, Descarreaux M, Hains F.Chronic shoulder pain of mypfascial origin: A randomized clinical trial using ischemic compression.J. Manip. Phyiol. Ther. 2010;33(5):362-369.

[26] Gulick DT, Palombaro K, Lattanzi JB. Effect of ischemic pressure using a Backnobber II device on discomfort associated with myofascial trigger points. J Bodyw Mov Ther. $2011 ; 15(3): 319-325$

[27] de Almeida Paz I, Kerppers II, Fréz AR. Effects of ischemic compression of trigger points in painful episodes of patients with chronic shoulder pain. Systematic Review. Man. Ther., Posturology Rehabil. J. 2014;12:172.

[28] Hanten WP, Olson SL, Butts NL, Nowicki AL. Effectiveness of a home program of ischemic pressure followed by sustained stretch for treatment of myofascial trigger points. Phys Ther.
2000;80(10):997-1003.

[29] Ganesh GS, Singh H, Mushtaq S, Mohanty P, Pattnaik M. Effect of cervical mobilization and ischemic compression therapy on contralateral cervical side flexion and pressure pain threshold in latent upper trapezius trigger points. J Bodyw Mov Ther. 2016;20(3):477-483.

[30] Cagnie B, Dewitte V, Coppieters I, Jessica Van Oosterwijck J.V, Cools A, Danneels L. Effect of ischemic compression on trigger points in the neck and shoulder muscles in office workers: a cohort study. J Manipulative Physiol Ther. 2013; 36(8):482-9.

[31] Kannan P.Management of Myofascial Pain of Upper Trapezius: A Three Group Comparison Study. Glob J Health Sci. 2015;4(5):46-52.

[32] Draper DO, Mahaffey C, Kaiser D, Eggett D, Jake J. Thermal ultrasound decreases tissue stiffness of trigger points in upper trapezius muscles. Physiother Theory Pract. 2010;26(3):167-172.

[33] Ilter L, Dilek B, Batmaz I,Ali-Ullah M, Sariyildiz M, Nas K, et al. Efficacy of pulsed and continuous therapeutic ultrasound in myofascial pain syndrome: A randomized controlled trial. Am. J. Phys. Med. Rehabil. 2015;94(7):547-554.

[34] Kavadar G, Calglar N, Ozen S, Demircioglu D. Efficacy of conventional uktrasound therapy on myofascial pain syndrome: A placebo controlled study. Agri. 2015; 27(4):190-196

[35] Cagnie B, Castelein B, pollie F, Stealannt L, Verhoeyen $\mathrm{H}$, Cools A. Evidence for the Use of Ischemic Compression and Dry Needling in the Management of Trigger Points of the Upper Trapezius in Patients with Neck Pain: A Systematic Review. Am J Phys Med Rehab. 2015;94(7):573-583

[36] Nambi GS, Sharma R, Inbasekaran D, Vaghesiya A, Bhatt $U$. Difference in effect between ischemic compression and muscle energy technique on upper trepezius myofascial trigger points: Comparative study. Int J Health Allied Sci. 2013;2(1):17 